\title{
Cone metric spaces and fixed point theorems of generalized T-Zamfirescu mappings
}

\author{
${ }^{1}$ A.K.Dubey, ${ }^{2}$ Rita Shukla, ${ }^{3}$ Ravi Prakash Dubey \\ ${ }^{1}$ Department of Mathematics, Bhilai Institute of Technology, Bhilai House, Durg, 491001, Chhattisgarh, INDIA \\ ${ }^{2}$ Department of Mathematics, Shri Shankracharya College of Engineering Bhilai, 490020 Chhattisgarh, INDIA \\ ${ }^{3}$ Department of Mathematics, Dr. C.V.Raman University, Bilaspur, Chhattisgarh, INDIA \\ *Corresponding author E-mail: anilkumardby@rediffmail.com
}

\begin{abstract}
In this paper, we obtain sufficient conditions for the existence of a unique common fixed point for pair of $\mathrm{T}$ Zamfirescu contraction mappings in the setting of complete cone metric spaces.
\end{abstract}

Keywords: Fixed point, complete cone metric space, T-Zamfirescu mapping, Subsequentially convergent.

\section{Introduction}

It is well known that the classic contraction mapping principle of Banach is a fundamental results in fixed point theory. Several authors have obtained various extensions and generalizations of Banach's theorem by considering contractive mappings on many different metric spaces. Recently, Guang and Xian [1] introduce the notion of cone metric spaces. He replaced real number system by ordered Banach space. He also gave the condition in the setting of cone metric spaces. These authors also described the convergence of sequences in the cone metric spaces and introduce the correspondig notion of completeness. The results in [1] were generalized by Sh.Rezapour and R. Hamlbarani [7] omitting the assumption of normality on the cone. Subsequently, many authors have generalized the results of Guang and Xian and have studied fixed point theorems for normal and non-normal cone, see for instance [9],[10],[11], [12], [15] and [16].

In 2009 [13], A. Beiranvand, S. Moradi, M. Omid and H. Pazandeh introduced new classes of contractive functions-T-Contraction and T-contractive mappings and then they established and extended the Banach contraction principle and the Edelstein's fixed point theorems. S. Moradi [4] introduce the T-Kannan contractive mapping which extend the well known Kannan's fixed point theorem given in [2]. In sequel, J.R. Morales and E Rojas [5] and [6] obtained sufficient conditions for the existence of a unique fixed point of T-Kannan contractive and T-contractive mappings respectively, on complete cone metric spaces.

In the present paper, we study the existence of a unique common fixed point for pair of T-Zamfirescu contraction mappings in the setting of complete cone metric spaces $(\mathrm{X}, \mathrm{d})$, generalizing consequently the results given in [1], [3] and [8].

\section{Preliminary notes}

First, we recall some standard notations and definitions in cone metric spaces with some of their properties (see $[1])$.

Definition 2.1 . Let E be a real Banach space and $\mathrm{P}$ a subset of E .P is called a cone if and only if : 
(i) $\mathrm{P}$ is closed, non-empty and $\mathrm{P} \neq\{0\}$,

(ii) $a x+b y \in P$ for all $x, y \in P$ and non-negative real numbers a,b;

(iii) $x \in P$ and $-x \in P \Rightarrow x=0 \Leftrightarrow P \cap(-P)=\{0\}$.

Given a cone $P \subset E$, we define a partial ordering $\leqslant$ on $\mathrm{E}$ with respect to $\mathrm{P}$ by $x \leqslant y$ if and only if $y-x \in P$. We shall write $x \ll y$ if $y-x \in \operatorname{int} P$, int $P$ denotes the interior of $\mathrm{P}$. The cone $\mathrm{P}$ is called normal if there is a number $K>0$ such that for all $x, y \in E$,

$0 \leqslant x \leqslant y$ implies $\|x\| \leqslant K\|y\|$

The least positive number $\mathrm{K}$ satisfying the above is called the normal constant of $\mathrm{P}$.

Definition 2.2. [1] Let $\mathrm{X}$ be a non-empty set and $d: X \times X \rightarrow E$ a mapping such that

$\left(d_{1}\right) 0 \leqslant d(x, y)$ for all $x, y \in X$ and $d(x, y)=0$ if and only if $x=y$;

$\left(d_{2}\right) d(x, y)=d(y, x)$ for all $x, y \in X$;

$\left(d_{3}\right) d(x, y) \leqslant d(x, z)+d(z, y)$ for all $x, y, z \in X$.

Then $\mathrm{d}$ is called a cone metric on $X$, and $(X, d)$ is called a cone metric space.

Example 2.3. Let $E=R^{2}, P=\{(x, y) \in E: x, y \geqslant o\}, X=R$ and $d: X \times X \rightarrow E$ defined by $d(x, y)=(\mid$ $x-y|, \alpha| x-y \mid)$, where $\alpha \geqslant 0$ is a constant. Then $(X, d)$ is a cone metric space .

Definition 2.4. (see [1]) Let $(X, d)$ be a cone metric space. Let $\left(x_{n}\right)$ be a sequence in $X$ and $x \in X$

(i) $\left(x_{n}\right)$ converges to $x$ if for every $c \in E$ with $0 \ll c$ there is an $n_{o}$ such that for all $n>n_{o}, d\left(x_{n}, x\right) \ll c$. We denote this by $\lim _{n \rightarrow \infty} x_{n}=x$ or $x_{n} \rightarrow x,(n \rightarrow \infty)$.

(ii) If for any $c \in E$ with $0 \ll c$ there is an $n_{o}$ such that for all $n, m \geqslant n_{o}, d\left(x_{n}, x_{m}\right) \ll c$, then $\left(x_{n}\right)$ is called a Cauchy sequence in $X$. Let $(X, d)$ be a cone metric space. If every Cauchy sequence is convergent in $X$, then $X$ is called a complete cone metric space.

Lemma 2.5.[1]) Let $(X, d)$ be a cone metric space, $P \subset E$ a normal cone with normal constant $K$. Let $\left(x_{n}\right),\left(y_{n}\right)$ be sequences in $X$ and $x, y \in X$.

(i) $\left(x_{n}\right)$ converges to $x$ if and only if $\lim _{n \rightarrow \infty} d\left(x_{n}, x\right)=0$;

(ii) If $\left(x_{n}\right)$ converges to $x$ and $\left(x_{n}\right)$ converges to $y$ then $x=y$. That is the limit of $\left(x_{n}\right)$ is unique;

(iii) If $\left(x_{n}\right)$ converges to $x$, then $\left(x_{n}\right)$ is Cauchy sequence;

(iv) $\left(x_{n}\right)$ is a Cauchy sequence if and only if $\lim _{n, m \rightarrow \infty} d\left(x_{n}, x_{m}\right)=0$;

(v) If $x_{n} \rightarrow x$ and $y_{n} \rightarrow y,(n \rightarrow \infty)$ then $d\left(x_{n}, y_{n}\right) \rightarrow d(x, y)$.

Definition 2.6(see[5]and[6]). Let $(X, d)$ be a cone metric space, $\mathrm{P}$ a normal cone with normal constant $\mathrm{K}$ and $T: X \rightarrow X$. Then

(i) $\mathrm{T}$ is said to be continuous if $\lim _{n \rightarrow \infty} x_{n}=x$, implies that $\lim _{n \rightarrow \infty} T\left(x_{n}\right)=T(x)$, for all $\left(x_{n}\right)$ in $\mathrm{X}$; 
(ii) $\mathrm{T}$ is said to be subsequentially convergent, if we have, for every sequence $\left(y_{n}\right)$ that $T\left(y_{n}\right)$ is convergent, implies $\left(y_{n}\right)$ has a onvergent subsequence;

(iii) $\mathrm{T}$ is said to be sequentially convergent, if we have, for every sequence $\left(y_{n}\right)$, if $T\left(y_{n}\right)$ is convergent, then $\left(y_{n}\right)$ is also convergent.

\section{Main results}

The results which we will give, are generalization of theorem 3.2 of [3]. First, we recall the following classes of contraction type mappings:

Definition 3.1[3] Let $(X, d)$ be a cone metric space and $T, S: X \rightarrow X$ two mappings

(i) The mapping $\mathrm{S}$ is called a T-Banach contraction, if there is $a \in[0,1)$ such that $d(T S x, T S y) \leqslant a d(T x, T y)$ for all $x, y \in X$.

(ii) A mapping $\mathrm{S}$ is called a T-Kannan contraction, if there is $b \in\left[0, \frac{1}{2}\right)$ such that $d(T S x, T S y) \leqslant b[d(T x, T S x)+$ $d(T y, T S y)]$ for all $x, y \in X$.

(iii) A mapping $\mathrm{S}$ is said to be a Chatterjea contraction, if there is $c \in\left[0, \frac{1}{2}\right)$ such that $d(T S x, T S y) \leqslant$ $c[d(T x, T S y)+d(T y, T S x)]$ for all $x, y \in X$.

It is clear that if we take $\mathrm{T}=\mathrm{I}_{d}$ (the identity map) in the Definition 3.1 we obtain the definitions of Banach contraction, Kannan mapping [2] and Chatterjea mapping [14].

Definition3.2[3] Let $(X, d)$ be a cone metric space and $T, S: X \rightarrow X$ two mappigs, $S$ is called a $T-Z$ amfirescu mapping (TZFS-mapping), if and only if, there are real numbers, $0 \leqslant a<1,0 \leqslant b, c<\frac{1}{2}$ such that for all $x, y \in X$, at least one of the next conditions are true;

(TZFS1): $d(T S x, T S y) \leqslant a d(T x, T y)$.

(TZFS2): $d(T S x, T S y) \leqslant b[d(T x, T S x)+d(T y, T S y)]$

(TZFS3): $d(T S x, T S y) \leqslant c[d(T x, T S y)+d(T y, T S x)]$.

If in Definition 3.2 we take $T=I_{d}$ (the identity map) and $E=\mathbb{R}_{+}$we obtain the definition of $T-Z$ amfirescu[8].

Lemma 3.3[3] Let $(X, d)$ be a cone metric space and $T, S: X \rightarrow X$ two mappings. If $S$ is a TZFS - mapping, then there is $0 \leqslant \delta<1$ such that

$d(T S x, T S y) \leqslant \delta d(T x, T y)+2 \delta d(T x, T S x)$ for all $x, y \in X$

Above inequality can be replace by $d(T S x, T S y) \leqslant \delta d(T x, T y)+2 \delta d(T x, T S y)$ for all $x, y \in X$. 
Theorem 3.4. Let $(X, d)$ be a complete cone metric space, $\mathrm{P}$ be a normal cone with normal constant K. Moreover, let $T: X \rightarrow X$. be a continuous and one to one mapping and $R, S: X \rightarrow X$ be a pair of T-Zamfirescu (TZFS) continuous mappings. Then

(i) For every $x_{o} \in X$,

$$
\lim _{n \rightarrow \infty} d\left(T R^{2 n+1} x_{o}, T R^{2 n+2} x_{o}\right)=0
$$

and

$$
\lim _{n \rightarrow \infty} d\left(T S^{2 n+2} x_{o}, T S^{2 n+3} x_{o}\right)=0 .
$$

(ii) There is $\vartheta \in X$ such that

$$
\lim _{n \rightarrow \infty} T R^{2 n+1} x_{o}=\vartheta=\lim _{n \rightarrow \infty} T S^{2 n+2} x_{o} .
$$

(iii) If $\mathrm{T}$ is subsequentially convergent, then $\left(R^{2 n+1} x_{o}\right)$ and $\left(S^{2 n+2} x_{o}\right)$ have a convergent subsequences.

(iv) There is unique common fixed point $u \in X$ such that $R u=u=S u$.

(v) If $\mathrm{T}$ is sequentially convergent, then for each $x_{o} \in X$ the iterate sequences $\left(R^{2 n+1} x_{o}\right)$ and $\left(S^{2 n+2} x_{o}\right)$ converge to $u$.

Proof: (i) Since R and S are pair of TZFS mapping, then by Lemma 3.3, there exists $0<\delta<1$ such that

$$
d(T R x, T S y) \leqslant \delta d(T x, T y)+2 \delta d(T x, T R x) \text { for all } x, y \in X
$$

Suppose $x_{o} \in X$ is an arbitrary point and the Picard iteration associated to R and S. $\left(x_{2 n+1}\right)$ and $\left(x_{2 n+2}\right)$ are defined by

$$
\begin{aligned}
& x_{2 n+2}=R x_{2 n+1}=R^{2 n+1} x_{o}, n=0,1,2, \ldots \\
& x_{2 n+3}=S x_{2 n+2}=S^{2 n+2} x_{o}, n=0,1,2, \ldots
\end{aligned}
$$

Thus,

$$
d\left(T R^{2 n+2} x_{o}, T R^{2 n+1} x_{o}\right) \leqslant h d\left(T R^{2 n+1} x_{o}, T R^{2 n} x_{o}\right)
$$

where $h=\frac{\delta}{1-2 \delta}<1$. Therefore, for all $n$ we have

$$
d\left(T R^{2 n+2} x_{o}, T R^{2 n+1} x_{o}\right) \leqslant h^{2 n+1} d\left(T R x_{o}, T x_{o}\right)
$$


Similarly, we have

$$
d\left(T S^{2 n+3} x_{o}, T S^{2 n+2} x_{o}\right) \leqslant k^{2 n+2} d\left(T S x_{o}, T x_{o}\right)
$$

From (1) and the fact the cone $\mathrm{P}$ is a normal cone we obtain that

$$
\left\|d\left(T R^{2 n+2} x_{o}, T R^{2 n+1} x_{o}\right)\right\| \leqslant K h^{2 n+1}\left\|d\left(T R x_{o}, T x_{o}\right)\right\|,
$$

taking limit $n \rightarrow \infty$ in the above inequality, we can conclude that

$$
\lim _{n \rightarrow \infty} d\left(T R^{2 n+2} x_{o}, T R^{2 n+1} x_{o}\right)=0
$$

Similarly, from (2) we have,

$$
\lim _{n \rightarrow \infty} d\left(T S^{2 n+3} x_{o}, T S^{2 n+2} x_{o}\right)=0
$$

(ii) Now, for $m, n \in N$ with $m>n$, we get

$$
\begin{aligned}
d\left(T R^{2 m+1} x_{o}, T R^{2 n+1} x_{o}\right) \leqslant & \left(h^{2 n+1}+\ldots+h^{2 m}\right) d\left(T R x_{o}, T x_{o}\right) \\
& \leqslant \frac{h^{2 n+1}}{1-h} d\left(T R x_{o}, T x_{o}\right)
\end{aligned}
$$

Again; as above, since $\mathrm{P}$ is a normal cone we obtain

$\lim _{n, m \rightarrow \infty} d\left(T R^{2 m+1} x_{o}, T R^{2 n+1} x_{o}\right)=0$.

Hence, the fact that $(X, d)$ is complete cone metric space, imply that $\left(T R^{2 n+1} x_{o}\right)$ is a Cauchy sequence in $X$, therefore there is $\vartheta \in X$ such that

$$
\lim _{n \rightarrow \infty} T R^{2 n+1} x_{o}=\vartheta .
$$

(iii) if $T$ is subsequentially convergent, $\left(R^{2 n+1} x_{o}\right)$ has a convergent subsequence. So there is $u \in X$ and $\left\{(2 n+1)_{i}\right\}_{i=1}^{\infty}$ such that

$$
\lim _{i \rightarrow \infty} R^{(2 n+1)_{i}} x_{o}=u .
$$

(iv) Since $\mathrm{T}$ and $\mathrm{R}$ are continuous mappings, we obtain;

$$
\lim _{i \rightarrow \infty} T R^{(2 n+1)_{i}} x_{o}=T u, \lim _{i \rightarrow \infty} T R^{(2 n+1)_{i}+1} x_{o}=T R u
$$

therefore, $T u=\vartheta=T R u$, and since $T$ is one-to-one, then $R u=u$. So $R$ has a fixed point.

Now, suppose that $R u=u$ and $R u_{1}=u_{1}$.

$$
\begin{aligned}
& d\left(T R u, T R u_{1}\right) \leqslant \delta d\left(T u, T u_{1}\right)+2 \delta d(T u, T R u) \\
& d\left(T u, T u_{1}\right) \leqslant \delta d\left(T u, T u_{1}\right)
\end{aligned}
$$

from the fact that $0 \leqslant \delta<1$ and that $T$ is one-to-one we obtain that $u=u_{1}$.

(v) It is clear that if $T$ is sequentially convergent, then for each $x_{o} \in X$, the iterate sequence $\left(R^{2 n+1} x_{o}\right) \operatorname{converges}$ to $u$. 
Similarly, we can prove that $\left(S^{2 n+2} x_{o}\right)$ converges to the fixed point of $S$ i.e $u$.

or $\lim _{i \rightarrow \infty} R^{(2 n+1)_{i}} x_{o}=u=\lim _{i \rightarrow \infty} S^{(2 n+2)_{i}} x_{o}$.

This completes the proof of the theorem.

\section{References}

[1] L.G.Huang, X.Zhang, Cone metric spaces and fixed point theorems of contractive mappings, J. Math. Anal. Appl.,332,(2007),1468-1476.

[2] R. Kannan, Some results on fixed points-II, Amer. Math.Monthly,76(1969)405-408.

[3] J.R. Morales and E. Rojas, T-Zamfirescu and T-Weak contraction mappings on cone metric spaces, arXiv:0909.1255v1[math.FA]7sep2009.

[4] S. Moradi, Kannan fixed point theorem on complete metric spaces and on generalized metric spaces depended on another function, arXiv:0903.1577v1[math.FA].

[5] J.R. Morales and E. Rojas, Cone metric spaces and fixed point theorems of T-Kannan contractive mappings, arXiv:0907.3949v1[math.FA].

[6] J.R.Morales and E. Rojas, Cone metric spaces and fixed point theorems of T-Contractive mappings, Revista Notas de Matematica, Vol4(2),No.269,2008,PP 66-78.

[7] Sh.Rezapour and R. Hamlbarani, Some notes on the paper "Cone metric spaces and fixed point theorems of contractive mappings", J. Math. Anal.Appl.,345(2), (2008),719-724.

[8] T.Zamfirescu, Fixed point theorems in metric spaces, Arch. Math.,23,(1972),292-298.

[9] D.Ilic and V. Rakocevic, Quasi-contraction on a cone metric space, Appl. Math. Lett.,22,(5),(2009),728-731.

[10] Z.Kadelburg, S.Radenovic and V.Rakocevic, Remarks on "Quasi-contraction on a cone metric space". Appl. Math.Lett.,(2009),doi:10.1016/j.aml.2009.06.003.

[11] M.S. Khan and M. Samanipour, Fixed point theorems for some discontinuous operators in cone metric space, Mathematica Moravica,Vol 12-2,(2008),29-34.

[12] V. Raja and S.M.Vaezpour, Some extension of Banach's contraction principle in complete cone metric spaces, Fixed point Theory and Applications, (2008), 11 P.

[13] A. Beiranvand, S.Moradi, M. Omid and H. Pazandeh, Two fixed point theorem for special mapping, arXiv;0903.1504v1[math.FA].

[14] S.K.Chatterjea, Fixed point theorems, C.R.Acad.Bulgare Sci.,25,(1972),727-730.

[15] A.K.Dubey and A. Narayan, Cone Metric Spaces and fixed point theorems for pair of contractive maps, Mathematica Aeterna, Vol 2, 2012, No.10,839-845.

[16] A.K.Dubey, Rita Shukla and R.P. Dubey, An extension of the paper "Cone metric spaces and fixed point theorems of contractive mappings" Int.J. of Appl. Mathematical Research, 2 (1) (2013), 84-90. 